\title{
Analyst
}

Cite this: Analyst, 2014, 139, 2000

\section{Detection of theophylline utilising portable electrochemical sensors}

\author{
Tiancheng Wang, Edward P. Randviir and Craig E. Banks* \\ The electrochemical oxidation of theophylline (TP) is investigated utilising screen-printed electrodes. \\ Through thorough investigation of $\mathrm{pH}$, we propose a reaction mechanism, finding that the oxidation of \\ TP is stable over a wide $\mathrm{pH}$ range, in particular under acidic conditions. Conversely under alkaline \\ conditions, theophylline fouls the electrode surface. The screen-printed carbon sensors are applied \\ towards the electroanalytical sensing of TP with a remarkable amount of success in aqueous solution at \\ physiological $\mathrm{pH}$. The screen-printed sensors have been shown to be applicable to the detection of TP \\ at unharmful, medicinally relevant $(55-110 \mu \mathrm{M})$, and toxic concentrations in aqueous media at \\ physiological $\mathrm{pH}$. Thus this work presents a proof-of-concept approach towards TP detection utilising \\ sensors commonly implemented in point-of-care applications.
}

Received 10th January 2014 Accepted 25th January 2014

DOI: $10.1039 / c 4 a n 00065$

www.rsc.org/analyst recognition proteins which make the electrochemistry complex. Surprisingly however, the direct oxidation of theophylline utilising SPEs appears to be overlooked in the literature. Hence, for the purpose of completeness we provide the electrochemical activities of three screen-printed sensors (edgeplane-like, basal-plane-like, and single-walled carbon nanotube SPEs) and compare to standard laboratory electrodes including gold, highly ordered pyrolytic graphite, glassy carbon, and boron-doped diamond.

\section{Experimental}

All chemicals were of the highest grade available, used without further purification, and obtained from Sigma Aldrich (UK). All aqueous solutions were made using deionised water of resistivity no less than $18.2 \mathrm{M} \Omega \mathrm{cm}$. Several buffer solutions were appropriately selected: $0.1 \mathrm{M}$ acetate buffer ( $\mathrm{pH} 4.0$ ); $0.1 \mathrm{M}$ Phosphate Buffer Solution (PBS) (pH 7.4); and $0.1 \mathrm{M}$ borate buffer (pH 11.5). For pH 1.0, 0.1 M sulphuric acid was utilised as the solvent. Theophylline stock solutions were prepared in each appropriate $\mathrm{pH}$ prior to testing. Voltammetric data were obtained utilising an Ivium CompactStat ${ }^{\mathrm{TM}}$ (Netherlands) potentiostat. SPEs (edge plane-like, termed ESPE and basal plane-like, termed BSPE) were fabricated in-house as described previously. ${ }^{14}$ Briefly, a relevant carbon ink formulation for efficient connection of the three electrodes was printed onto a flexible polyester film and cured in an oven at $60{ }^{\circ} \mathrm{C}$ for 30 minutes. Next a silver/silver chloride reference electrode was included by screen-printing $\mathrm{Ag} / \mathrm{AgCl}$ paste and subsequently curing at $60{ }^{\circ} \mathrm{C}$ for 30 minutes. Finally, a dielectric paste was printed on top to define the electrodes $(3 \mathrm{~mm}$ working electrode) and protect the connections. Further information regarding the inks can be found in ref. 15. The single-walled 
carbon nanotube SPEs were made (termed SW-SPE) as above, except that commercially available single-walled carbon nanotube ink was printed over the working electrode only following the third curing procedure. ${ }^{16}$ Conventional electrodes $(3 \mathrm{~mm}$ Glassy Carbon (GC), 4.9 mm Edge Plane and Basal Plane Pyrolytic Graphite (EPPG/BPPG), $3 \mathrm{~mm}$ gold, $3 \mathrm{~mm}$ Boron Doped Diamond (BDD)) were obtained commercially from Le Carbone Ltd., Sussex, U.K. and were polished to a mirror-like shine utilising diamond spray of decreasing particle size. A saturated calomel electrode (SCE) was utilised as a reference and a platinum wire as an auxiliary electrode, unless otherwise stated.

\section{Results and discussion}

\section{Electrode investigation}

Attention is first turned towards the electrochemical response of $1 \mathrm{mM}$ theophylline at several electrode substrates at physiological pH. Fig. 1 compares all electrode substrates investigated. Inspection of Fig. 1 reveals a general trend where the peak potential decreases as the peak current density increases; this is particularly evident for the case of electrodes such as EPPG which exhibits a peak potential of $+1.00 \mathrm{~V}$ and an average peak current of $191 \mu \mathrm{A} \mathrm{cm}^{-2}(\% \mathrm{RSD}=5 \% ; N=3)$. Conversely, by taking the gold electrode as an example, a large overpotential $(+1.17 \mathrm{~V})$ is required to drive the electrochemical oxidation of TP which compromises on both the average observed current density of $57 \mu \mathrm{A} \mathrm{cm}^{-2}$ and the reproducibility ( $\% \mathrm{RSD}=46 \% ; N=3$ ). Turning attention to the screen-printed electrodes, we investigate a trio of SPEs which are electrochemically divergent. Fig. 1 shows the edge plane-like SPE (ESPE) and the single-walled carbon nanotube SPE (SW-SPE) exhibiting similar behaviour, with peak potentials corresponding to $+1.04 \mathrm{~V}$ and $+1.03 \mathrm{~V}$, respectively.

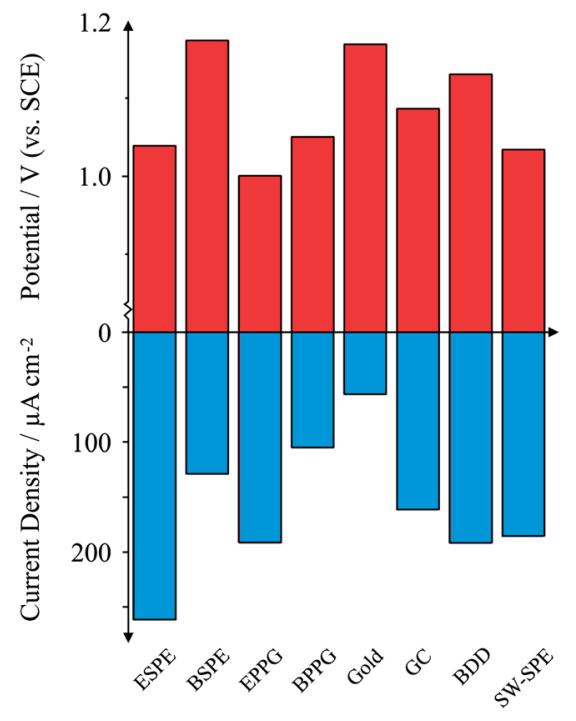

Fig. 1 Graphical representation of peak current densities (blue) and peak potentials (red) for the series of electrodes utilised within this work. All experiments were conducted using $1 \mathrm{mM}$ TP in $0.1 \mathrm{M}$ PBS $\left(\mathrm{pH}\right.$ 7.4). Scan rate: $100 \mathrm{mV} \mathrm{s}^{-1}$.
Similar reactivities are likely due to the edge-plane content of the respective electrodes. Remarkably, the ESPE exhibits the highest peak current density of all electrodes studied here, corresponding to an average value of $262 \mu \mathrm{A} \mathrm{cm} \mathrm{cm}^{-2}$ and furthermore exhibiting a \% RSD of $5 \%(N=3)$; such an electrode could prove to be useful for electroanalytical sensing of TP. Conversely, the basal plane-like SPE (BSPE) exhibits a high peak potential $(+1.17 \mathrm{~V})$ and a low peak current density of $129 \mu \mathrm{A} \mathrm{cm}^{-2}$, similar to the case of the gold and basal plane pyrolytic graphite (BPPG) electrodes. Though the observed current densities are not as high for BSPE and SWSPE as that observed for EPPG, they still exhibit a magnitude and reproducibility $(<5 \%)$ which are useful for electroanalytical sensing. Glassy Carbon (GC) and Boron Doped Diamond (BDD) both exhibit high peak potentials and low currents. We continue to investigate the edge-plane-like SPE in terms of $\mathrm{pH}$ and electroanalytical capabilities; the SW-SPE could potentially be also used but we see no further benefits of the carbon nanotubes in this instance, generally due to cost implications.

\section{pH}

To optimise the electroanalytical conditions it is necessary to investigate the effect of $\mathrm{pH}$ on the observed peak potentials and currents for TP. Fig. 2 depicts voltammetric profiles of $1 \mathrm{mM}$ TP, corresponding to changes in $\mathrm{pH}$. One can immediately observe that the peak potentials increase as the $\mathrm{pH}$ decreases (inset), yet the relative magnitude of the peak current fluctuates, indicating that the efficiency of electron transfer for TP is $\mathrm{pH}$ dependent. The plot of peak potential versus $\mathrm{pH}$, presented in the inset of Fig. 2, illustrates the $\mathrm{pH}$ dependence of peak potential for this system which is linear from $\mathrm{pH} 1$ to 8.5 , beyond which there is deviation; this is in agreement with its $\mathrm{p} K_{\mathrm{a}}$, reported to be $\sim 8.6 .{ }^{17}$ The slope of the graph inset in Fig. 2 corresponds to 61.7 $\mathrm{mV} \mathrm{pH}^{-1}\left(R^{2}=0.994\right)$, corresponding to approximately an equal number of protons and electrons transferred. Above $\mathrm{pH}$ 8.5, a secondary wave appears (not reported), masking the analytical peak. We believe further peaks to be a result of electrode fouling, which was apparent under strongly alkaline conditions. Given the lack in change of mechanism over the large $\mathrm{pH}$ range prior to the $\mathrm{p} K_{\mathrm{a}}$, we consequently select physiological $\mathrm{pH}$ for electroanalysis which is presented later.

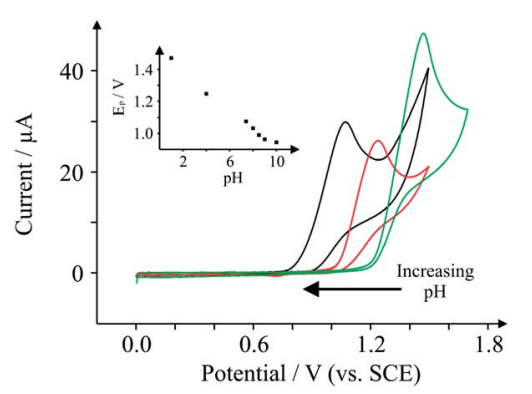

Fig. 2 Cyclic voltammetric profiles obtained for $1 \mathrm{mM}$ TP using an ESPE working electrode under various conditions: $\mathrm{pH} 1.0$ (green); $\mathrm{pH}$ 4.2 (red); and $\mathrm{pH} 7.4$ (black). Scan rate: $100 \mathrm{mV} \mathrm{s}^{-1}$. Inset: peak potential versus $\mathrm{pH}$ for the entire $\mathrm{pH}$ range utilised. 


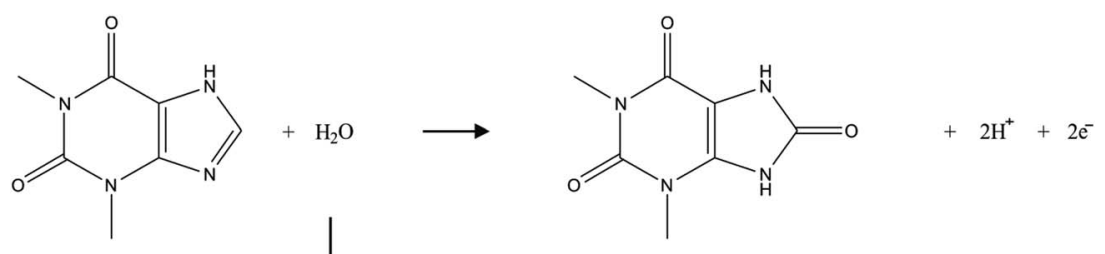

Theophylline
The mechanism of TP oxidation is next considered by taking into account the $\mathrm{pH}$. The structure of TP is given in Scheme 1, alongside two proposed reaction mechanisms. Given that TP has a structure which can be thought of as analogous to guanine, the proposed mechanism involves a two electron two proton oxidation of the aromatic carbon sandwiched between two electron-withdrawing nitrogen heteroatoms, as is in the case of the structurally similar guanine. ${ }^{18}$ Of course, under highly basic conditions the oxidation of the nitrogen heteroatom to a hydroxylamine is possible in small quantities, yet we believe that the formation of the carbonyl is more likely. The fact that the mechanism does not change dramatically above the $\mathrm{p} K_{\mathrm{a}}$ acts as support for this mechanism.

\section{Electroanalytical detection of theophylline}

To demonstrate the applicability of SPEs to TP, we investigate the effect of peak currents with respect to the concentration of TP present within a buffer solution at physiological pH. Fig. 3 depicts the effect of peak current as the concentration of TP in pH 7.4 PBS increases. The electroanalytical peak observed at $c a$. $+1.0 \mathrm{~V}$ increases linearly as a function of TP concentration, as depicted in the inset of Fig. 3: $I_{\mathrm{p}} / \mu_{\mathrm{A}}=12.28 \mu \mathrm{A} \mathrm{mM}{ }^{-1}+0.91 \mu \mathrm{A}$;

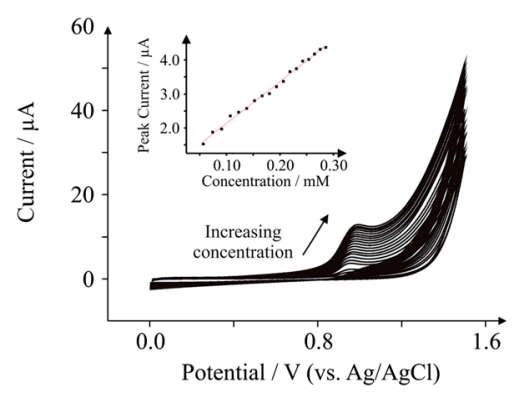

Fig. 3 Electroanalytical cyclic voltammetric profiles of TP utilising an ESPE working electrode. The on-board carbon auxiliary and $\mathrm{Ag} / \mathrm{AgCl}$ reference electrode is utilised in this instance. Scan rate: $100 \mathrm{mV} \mathrm{s}^{-1}$.
$N=18 ; R^{2}=0.994$, with a limit of detection of $10 \mu \mathrm{M}$. The concentrations studied here range from 50-290 $\mu \mathrm{M}$, which falls within the safe concentrations found in the plasma of medicinal TP users (55-110 $\mu \mathrm{M}$ - anything higher is considered dangerous), ${ }^{19}$ indicating that our protocol is potentially acceptable for TP detection in plasma for clinical screening applications. Furthermore the sensors exhibit \% RSD values of no more than $5 \%(N=3)$ which is within the constraints of acceptable (electro)analytical chemistry.

\section{Conclusions}

The detection of TP utilising SPEs has been described. Screenprinted sensors such as SW-SPE and BSPE replicate standard macroelectrodes such as EPPG with a relatively small loss of peak current density and increase in peak potential. Compared to other working electrodes such as gold, BPPG and GC, all the SPEs are superior in terms of peak current density and peak potential. ESPE however outperforms EPPG in terms of peak current density and thus is more suitable than EPPG for electroanalytical sensing of TP. SPEs also carry the added benefit of application to point-of-care systems. The SPEs are shown to be applicable to the detection of TP at both acidic and physiological $\mathrm{pH}$, yet display unstable voltammetric waves under highly alkaline conditions. A two proton two electron mechanism is proposed for the electrochemical oxidation of TP as a result of the $\mathrm{pH}$ investigations. The electrodes also display a remarkably high level of electroanalytical linearity at physiological pH. In the future, such electrodes need to be investigated further towards medicinal formulations containing TP, as well as plasma samples for screening purposes. Additionally, exploring the possibility of screen-printed microbands and arrays could prove to be important as the improved current densities produced by such sensing platforms could prove to be beneficial for electroanalytical applications. Such work, in addition to previous work regarding SPEs, highlights the range of opportunities for such technologies and as a result we believe the possibilities for SPE application are essentially 
limitless in terms of electrochemically screening molecules for point-of-care applications.

\section{References}

1 P. J. Barnes and R. A. Pauwels, Eur. Respir. J., 1994, 7, 579-591.

2 A. H. Dawson and I. M. Whyte, Br. J. Clin. Pharmacol., 1999, 48, 278-283.

3 M. Shannon, Ann. Intern. Med., 1993, 119, 1161-1167.

4 C. E. Cook, M. E. Twine, M. Myers, E. Amerson, J. A. Kepler and G. F. Taylor, Res. Commun. Chem. Pathol. Pharmacol., 1976, 13, 497-505.

5 B. Srdjenovic, V. Djordjevic-Milic, N. Grujic, R. Injac and Z. Lepojevic, J. Chromatogr. Sci., 2008, 46, 144-149.

6 M. E. Jolley, J. Anal. Toxicol., 1981, 5, 236-240.

7 E. E. Ferapontova, E. M. Olsen and K. V. Gothelf, J. Am. Chem. Soc., 2008, 130, 4256-4258.

8 Y.-H. Zhu, Z.-L. Zhang and D.-W. Pang, J. Electroanal. Chem., 2005, 581, 303-309.

9 N. Spãtaru, B. V. Sarada, D. A. Tryk and A. Fujishima, Electroanalysis, 2002, 14, 721-728.
10 Y. V. Ulyanova, A. E. Blackwell and S. D. Minteer, Analyst, 2006, 131, 257-261.

11 B. H. Hansen and G. Dryhurst, J. Electroanal. Chem. Interfacial Electrochem., 1971, 32, 405-414.

12 N. C. Foulds, J. M. Wilshere and M. J. Green, Anal. Chim. Acta, 1990, 229, 57-62.

13 K. S. Lee, T.-H. Kim, M.-C. Shin, W.-Y. Lee and J.-K. Park, Anal. Chim. Acta, 1999, 380, 17-26.

14 N. A. Choudry, D. K. Kampouris, R. O. Kadara and C. E. Banks, Electrochem. Commun., 2010, 12, 6-9.

15 E. P. Randviir, D. A. C. Brownson, J. P. Metters, R. O. Kadara and C. E. Banks, Phys. Chem. Chem. Phys., 2014, 16, 45984611.

16 E. P. Randviir, J. P. Metters, J. Stainton and C. E. Banks, Analyst, 2013, 138, 2970-2981.

17 A. V. Trask, W. D. S. Motherwell and W. Jones, Int. J. Pharm., 2006, 320, 114-123.

18 R. N. Goyal, B. K. Puri and N. Jain, J. Chem. Soc., Perkin Trans. 2, 2001, 832-837.

19 P. A. Mitenko and R. I. Ogilvie, N. Engl. J. Med., 1973, 289, 600-603. 